\title{
Vulnerabilidad hídrica de la cuenca del río blanco, en el municipio de la calera, considerando los escenarios de cambio climático propuestos por la corporación autónoma regional de cundinamarca - car
}

\author{
HYDRIC VULNERABILITY OF BLANCO RIVER BASIN, IN THE
}

MUNICIPALITY OF LA CALERA, CONSIDERING THE CLIMATE

CHANGE SCENARIOS PROPOSED BY THE REGIONAL AUTONOMOUS

CORPORATION OF CUNDINAMARCA - CAR

\author{
${ }^{1}$ Laura Johanna Rojas Garzón, ${ }^{2}$ Víctor Leonardo López Jiménez \\ ${ }^{1,2}$ Facultad de Ingeniería, Universidad de La Salle, Bogotá, Colombia. \\ ${ }^{1}$ laurajohannarojgar@gmail.com, orcid.org/0000-0001-7273-014X \\ ²vlopez@unisalle.edu.co, orcid.org/0000-0001-6341-3062
}

Recibido: 03/09/2016 • Aprobado: 30/10/2016

\section{Resumen}

En este estudio se analizó la vulnerabilidad hidrica de la cuenca del río Blanco, para las veredas Treinta y Seis, Jerusalén, La Junia y Tunjaque del municipio de La Calera, considerando los escenarios de cambio climático propuestos para la jurisdicción de la Corporación Autónoma Regional de Cundinamarca CAR (A2 y B2 periodo 2011-2040), bajo escenarios de emisiones AR4 del IPCC (2007). La metodología utilizada permitió caracterizar los componentes físicos, socio-económicos y ambientales de las veredas en estudio, para posteriormente identificar y evaluar los factores de riesgo que los acompañan, en especial aquellos relacionados con el posible desabastecimiento hídrico. Tras el análisis, se concluyó que el riesgo actual de sequia, degradación de suelos y remoción en masa se incrementará, situándose en un nivel medio alto debido a las nuevas condiciones climáticas que plantea el calentamiento global.

Palabras clave: cambio climático, escenarios de emisiones, variabilidad climática, vulnerabilidad hídrica.

\begin{abstract}
In this study, the water vulnerability of the Blanco River basin was analyzed for the rural areas of Treinta y Seis, Jerusalén, La Junia and Tunjaque, of the municipality of La Calera, considering climate change scenarios proposed by the Regional Autonomous Corporation of Cundinamarca CAR (A2 and B2 period 2011-2040), under Ipcc emission scenarios
\end{abstract}


Vulnerabilidad hídrica de la cuenca del río Blanco, en el municipio de La Calera, considerando los escenarios de cambio climático propuestos por la Corporación Autónoma Regional de Cundinamarca - CAR

AR4 (2007). The methodology used allowed to characterize the physical, socio-economic and environmental components of the rural areas under study, to later identify and evaluate the risk factors that accompany them, especially those related to possible water shortages. After this analysis, it was concluded that the current risk of drought, soil degradation and mass removal will increase to a medium high level, due to the new climatic conditions of global warming.

Key words: climate change, climate variability, emissions scenarios, hydric vulnerability.

\section{Introducción}

El recurso hídrico, como eje central en el desarrollo de la vida y como principal regulador de los ecosistemas, está sometido al impacto que pueden causar los eventos de origen natural y los derivados de las actividades antrópicas, al igual que lo está a los generados por el cambio climático y la variabilidad climática. Algunas de estas actividades están asociadas a la agricultura y los cambios en el uso del suelo; construcción y gestión de embalses; emisiones de sustancias contaminantes y tratamiento de aguas residuales (Bates et al. 2008). El impacto generado por los eventos mencionados (de origen natural o socio-natural) y por las actividades antrópicas puede modificar el régimen hidrológico de un territorio, así como las condiciones de calidad, cantidad y uso del agua.

En la contribución del grupo de trabajo I al Quinto Informe de Evaluación del Ipcc - Grupo Intergubernamental de Expertos sobre el Cambio Climático, se afirma que el calentamiento en el sistema climático global es inequívoco y que desde la década de 1950 muchos de los cambios observados no han tenido precedentes en los últimos decenios a milenios (Ipcc 2013). Además, enuncia que una de las consecuencias del calentamiento global serán los cambios en el ciclo del agua, los cuales se han evidenciado en las variaciones del contenido de vapor de agua de la atmósfera y en la distribución de la salinidad de los océanos. En consecuencia, se espera que haya cambios de frecuencia y/o intensidad de fenómenos meteorológicos extremos (Ipcc 2013), como la posible prolongación de los períodos de sequía y la alteración de los valores extremos de la precipitación (cambios sujetos a una variabilidad inter-decadal como la relacionada con el Evento El Niño-La Niña - Enos, por ejemplo), los cuales están asociados muchas veces a la ocurrencia de desastres.

El impacto del cambio climático, sumado a la variabilidad climática natural y a los eventos catastróficos de origen natural y socio-natural, pueden modificar el régimen hidrológico actual de un territorio y las condiciones de calidad y uso del agua. Por lo anterior, en el presente estudio se buscó analizar el impacto sobre el recurso hídrico ante los efectos del cambio climático en la cuenca río Blanco - sector norte, jurisdicción de la Corporación Autónoma Regional de Cundinamarca CAR, específicamente en las veredas Treinta y Seis, Jerusalén, La Junia y Tunjaque, del municipio de La Calera. Este análisis se llevó a cabo tomando como tema central la identificación y evaluación del riesgo de desastre, para reducir la vulnerabilidad a los fenómenos hidrometeorológicos extremos y aumentar la resiliencia frente a los riesgos que no pueden eliminarse (Ipcc 2012).

\section{Metodología}

A partir de información de primera mano, recopilada por medio de encuestas y por información secundaria, se llevó a cabo la caracterización física, social y económica de la zona comprendida por las veredas en estudio. Posteriormente se realizó la caracterización climática, no sin antes realizar un juicioso análisis de complementación de series y homogenización de las mismas, definiendo como periodo de estudio 1997 a 2012, debido a la limitada disponibilidad de datos en el área de estudio. Los parámetros precipitación y temperatura fueron regionalizados por medio de la 
herramienta ArcGIS. Paso siguiente, se determinó el balance hídrico por medio de la ecuación simplificada propuesta en el Estudio Nacional del Agua 2010, de escorrentía igual a la precipitación menos la evapotranspiración real (ESC=P-ETR). Esta ecuación considera que los cambios de almacenamiento tienden a minimizarse y pueden suponerse nulos para cualquier masa de agua, en áreas extensas y en largos periodos (Ideam 2010). De otro lado, se utilizó la ecuación de Budyco para determinar los valores de la evapotranspiración real. Es importante anotar que, el balance hídrico se realizó para la microcuenca río Blanco, tomando como punto de cierre la estación Choachí- El Uval.

Con el fin de determinar las anomalías en la series de tiempo se analizó la precipitación, comparando la media mensual multianual con los valores históricos cuyas oscilaciones (por encima o por debajo de la media mensual multianual), en su mayoría, coincidieron con los periodos identificados por Montealegre en su estudio sobre el Enos en Bogotá D.C. (Montealegre 2012) para el departamento de Cundinamarca en los que se presentó el fenómeno Enos: La Niña (1999-2000, 2007-2008, 2010-2011) y El Niño (1997-1998, 2002, 2006, 20092010). Se encontró, además, que algunas fluctuaciones por debajo o por encima de la normal climática corresponden a temporadas secas o lluviosas anuales, por lo que no son consideradas como efectos de eventos Enos.

Siguiendo la metodología propuesta por López V. citado por Arango en su trabajo sobre la identificación y análisis de los factores de riesgo en el área de la cuenca alta del río Bogotá (Arango 2012), para determinar el riesgo al que está sometida la población del área de estudio a la ocurrencia de eventos catastróficos, se evaluó la amenaza (factor externo) y se realizó el análisis de la vulnerabilidad (factor interno) para, finalmente, establecer con la relación de estos el nivel de riesgo del sistema, utilizando la ecuación 1.

$$
\mathrm{R}=\mathrm{f}(\mathrm{A} \times \mathrm{VG})
$$

Dónde: $\mathrm{A}=$ Amenaza

$\mathrm{VG}=$ Vulnerabilidad Global

El valor del riesgo actual para la zona de estudio se determinó considerando los rangos establecidos para la calificación de la amenaza (entre 1,0 y 10,0) y la vulnerabilidad (entre 0,1 y 1,0 ). De esta manera el tope máximo de la escala del riesgo se estableció en 10 , como se puede ver en Tabla 1.

La evaluación de las amenazas se realizó utilizando indicadores, creados para aquellos eventos que tuvieran relación con el desabastecimiento hídrico: incendio forestal, degradación de suelos - corrimiento de la frontera agrícola y remoción en masa. En cuanto a la evaluación del evento de sequía se utilizaron los índices de precipitación estandarizada (SPI, por sus siglas en inglés) (OMM 2012), el de precipitación (I) (López 2006) y el de la variación porcentual promedio de precipitación, todos para los periodos en los que hubo ocurrencia del fenómeno El Niño.

TABLA 1

Rango de calificación del riego, amenaza y vulnerabilidad

\begin{tabular}{|c|c|c|c|c|c|c|c|c|}
\hline \multicolumn{3}{|c|}{ Amenaza } & \multicolumn{3}{|c|}{ Vulnerabilidad } & \multicolumn{3}{|c|}{ Riesgo } \\
\hline \multicolumn{2}{|c|}{ Nivel } & Rango & \multicolumn{2}{|l|}{ Nivel } & Rango & \multicolumn{2}{|c|}{ Nivel } & Rango \\
\hline \multirow{2}{*}{ Alta } & \multirow{2}{*}{ A } & \multirow{2}{*}{$7,0-10,0$} & \multirow{2}{*}{ Alta } & \multirow{2}{*}{ A } & \multirow{2}{*}{$0,8-1,0$} & Muy Alto & MA & $8,1-10,0$ \\
\hline & & & & & & Alto & A & $6,1-8,0$ \\
\hline Media & M & $4,0-6,9$ & Media Alta & $\mathrm{Ma}$ & $0,6-0,7$ & Medio Alto & $\mathrm{Ma}$ & $4,1-6,0$ \\
\hline \multirow{2}{*}{ Baja } & \multirow{2}{*}{ B } & \multirow{2}{*}{$1,0-3,9$} & Media & M & $0,4-0,5$ & Medio & $\mathrm{M}$ & $2,6-4,0$ \\
\hline & & & Baja & B & $0,1-0,3$ & Bajo & B & $1,0-2,5$ \\
\hline
\end{tabular}

Fuente: modificado de Arango 2012. 
Debido a la limitada disponibilidad de información hidrometeorológica en la cuenca río Blanco, no se utilizaron índices como el de estrés hídrico (Rwsi), el de retención y regulación hídrica (IRH), el de extracción de agua subterránea (Ieas), el integral de uso de agua (Iiua) y el de vulnerabilidad por desabastecimiento hídrico (IVH). Es de anotar que, para cuencas con mayor información estos índices pueden dar una visión más amplia de las condiciones del sistema hídrico natural y la presión al recurso por la intervención antrópica.

La determinación de la vulnerabilidad del recurso hídrico en la cuenca se realizó considerando el enfoque de vulnerabilidad global propuesto por WilchesChaux (Wilches-Chaux 1989) y se tuvio en cuenta los distintos componentes del sistema (físico, socio-económico y ambiental) con el fin de hallar los efectos sobre la disponibilidad del recurso en los distintos escenarios de riesgo. En la ecuación 2 se muestra la relación utilizada para la determinación de la vulnerabilidad hídrica global, en la cual se entrega un peso del $60 \%$ a la vulnerabilidad total (VT) y del $40 \%$ a la vulnerabilidad derivada de los recursos (VR)

$$
V G=[(V T \times 0,6)+(V R \times 0,4)]
$$

La vulnerabilidad total está compuesta por seis componentes que son: ambiental (FVA), social (FVS), económico (FVE), político-institucional (Fvpi) y físicoestructural (Fvfe), las cuales a su vez están compuestas por diferentes factores que fueron valorados por medio del uso de indicadores. Considerando que no todos los componentes son igualmente importantes al momento de establecer el nivel de riesgo que tiene el sistema (la cuenca, en este caso) frente a la posible ocurrencia de uno de los eventos identificados, se hizo una ponderación del valor de la vulnerabilidad de cada uno de estos como se muestra en la siguiente ecuación:

$$
\begin{aligned}
\mathrm{VT}= & {[(\mathrm{FVS} \times 0,3)+(\mathrm{FVA} \times 0,3)+(\mathrm{FVFE} \times 0,2)+} \\
& (\mathrm{FVE} \times 0,1)+(\mathrm{FVPI} \times 0,1)]
\end{aligned}
$$

Al momento de incluir el cambio climático como modificador del riesgo actual del recurso hídrico en las veredas que conforman el área en estudio, se siguió la metodología propuesta por Guerrero y Saavedra (Guerrero \& 2013), empleando un factor de ponderación de cambio climático (Fpcc), el cual está ligado a las proyecciones del comportamiento de la precipitación y la temperatura descritos por Pabón (Pabón 2011) para la jurisdicción CAR, lo mismo que a las condiciones antrópicas propias de la región y a las características económicas, demográficas, de gobernanza y tecnología, para el periodo 2011-2040, bajo los escenarios de emisión SRES (por sus siglas en ingles) de cambio climático del Cuarto Informe de Evaluación del Ipcc AR4 (2007) (Ipcc 2007), vigentes para el momento en que se desarrolló esta investigación; específicamente se emplearon los escenarios A2 y B2, a la escala regional trabajada por Pabón (2011) para la jurisdicción CAR (Pabón 2011).

El Fpcc se estableció como posible variación (aumento o disminución probable) en un rango de hasta el $20 \%$ sobre los valores reales actuales de riesgo en la región (Guerrero \& S 2013) El Fpcc se evalúa bajo dos enfoques: líneas argumentales de escenarios de cambio climático e indicadores sobre aspectos climáticos y antrópicos ligados al cambio, para eventos asociados a bajas y altas precipitaciones; la descripción y calificación del riesgo futuro, teniendo en cuenta las características físicas del territorio y los enfoques utilizados en el diseño de los indicadores.

\section{Resultados y discusión}

A pesar que la macro-región climática circundante del área de estudio posee un régimen bimodal, determinado por el doble paso de la Zona de Confluencia Intertropical (Zcit) en los dos semestres del año, en esta zona no se presenta un descenso significativo de la precipitación ni de la humedad a mediados del año, debido a la influencia de los vientos húmedos provenientes de los Llanos Orientales y de la Amazonía condición que marca una tendencia monomodal en el comportamiento del clima. Puede decirse, entonces, que la región en la que se encuentran las veredas Treinta y Seis, Jerusalén, La Junia y Tunjaque, del municipio de La Calera, está sometida a un régimen climático en el que los mayores valores de precipitación y humedad se 
registran entre abril y agosto, siendo junio el mes más húmedo, y un periodo de estiaje que se inicia en el mes de diciembre y va hasta mediados de marzo.

La precipitación del área de estudio oscila entre 1100 y $1400 \mathrm{~mm}$ anuales. En cuanto a la temperatura, esta presenta valores medios entre $16^{\circ} \mathrm{C}$ y $6^{\circ} \mathrm{C}$, disminuyendo de forma gradual hacia la alta montańa, específicamente hacia la zona donde se encuentra la Peńa de Tunjaque. El cálculo del balance hídrico, muestra la relación entre precipitación y ETR, en la cual se puede ver que los volúmenes de lluvia superan las cantidades evapotranspiradas en la mayor parte del año, a excepción de diciembre, enero y febrero, los cuales corresponden a los meses secos en la región, con un déficit de precipitación en el periodo de $45,7 \mathrm{~mm}$, siendo el mes de enero el de mayor déficit $(21,07 \mathrm{~mm})$. Como un dato adicional se destaca que, durante el periodo lluvioso o de mayor humedad (marzo a noviembre), el mes de mayo es el que presenta los mayores valores medios de escorrentía con 91,42 mm.
Por medio del análisis de alteraciones relacionadas con la variabilidad climática, se pudo comprobar que la señal de variabilidad interanual El Niño-La Niña-Oscilación del Sur (Enos) para el periodo 1997 - 2012 en el área de estudio, se ve reflejada en déficit y excesos de precipitación. Como se muestra en la Fig. 1, cuando El Niño es precedido o sucedido por períodos de bajas precipitaciones los eventos de sequía se intensifican en la cuenca; de manera similar, cuando La Nińa es precedida o sucedida por períodos lluviosos, se intensifican los eventos de remoción en masa y las inundaciones.

En cuanto al cálculo de la variación porcentual promedio para cada evento se identificaron los eventos más intensos para la cuenca río Blanco: El Niño 1997 - 1998 con una reducción de lluvia de 32\% con respecto a la precipitación anual promedio; y La Niña 2010-2011 con un aumento en la precipitación promedio de $22,5 \%$.

\begin{tabular}{|c|c|c|c|c|c|c|c|c|c|c|c|c|c|c|c|c|c|c|c|c|c|c|c|c|}
\hline \multirow{3}{*}{$\begin{array}{l}\text { UBICACIÓN } \\
\text { HISTÓRICA } \\
\text { DEL EVENTO }\end{array}$} & \multicolumn{24}{|c|}{ DURACIÓN DEL ENOS } \\
\hline & \multicolumn{12}{|c|}{ AÑO 1} & \multicolumn{12}{|c|}{ AÑO 2} \\
\hline & ENE & FEB & MAR & $\mathrm{ABR}$ & MAY & JUN & JUL & AGO & SEP & OCT & NOV & DIC & ENE & FEB & MAR & $\mathrm{ABR}$ & MAY & $\pi$ UN & JL & AGO & SEP & OCT & Nov & DIC \\
\hline
\end{tabular}

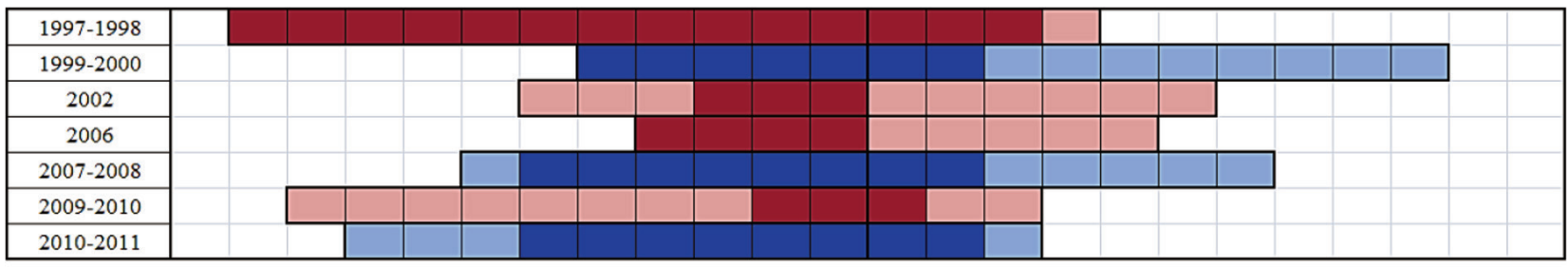

Fig. 1. Periodos en los que se identifican anomalías de precipitación relacionadas con El Niño y La Niña en la cuenca río Blanco.

Fuente: elaboración propia con base en las épocas de ocurrencia de los fenómenos El Niño y La Niña de acuerdo a las anomalías de la temperatura superficial del mar (TSM) registradas en el océano Pacífico central - Región Niño 3 (Montealegre 2012) y el análisis de alteraciones por variabilidad climática de la zona de estudio. Las áreas oscuras corresponden a los meses en que son más notorias las anomalías en la precipitación.

Uno de los objetivos del estudio es la identificación y análisis de eventos amenazantes que afecten la disponibilidad del recurso hídrico en la zona de estudio; estos eventos están relacionados con las épocas de sequía y las de abundantes precipitaciones que acompañan la estacionalidad, entendida como la distribución espacio temporal a lo largo del año, así como a la variabilidad climática ligada a la ocurrencia y características del evento Enos. El proceso de identificación de los eventos potencialmente catastróficos dio como resultado que las amenazas más representativas son la sequía y la remoción en masa. Al respecto, es bueno resaltar que la probabilidad de ocurrencia de incendios forestales y la degradación de los suelos, aunque su origen sea más antrópico que natural, se incrementa durante los extensos periodos de sequía, 
Vulnerabilidad hídrica de la cuenca del río Blanco, en el municipio de La Calera, considerando los escenarios de cambio climático propuestos por la Corporación Autónoma Regional de Cundinamarca - CAR

ligados muchas veces a la presencia del fenómeno de El Niño; de otro lado, la remoción en masa, es más frecuente durante las épocas de fuertes lluvias como las que acompańan el fenómeno de La Niña. En el estudio se incluyó el sismo para ser analizado como un evento que, a pesar de no tener una relación directa con la variabilidad y el cambio climático, su ocurrencia podría generar daños en la red de distribución (como en efecto ocurre en la mayoría de los casos) y/o potenciar eventos como los deslizamientos que, como ya se anotó, podrían afectar la calidad y el suministro del recurso.

Tabla 2

Índices e indicadores para la evaluación de amenazas

\begin{tabular}{|c|c|c|c|}
\hline \multicolumn{2}{|c|}{ Amenaza } & Índices e indicadores utilizados & Valor medio de amenaza \\
\hline \multirow{3}{*}{$\begin{array}{l}\text { Eventos asociados a las } \\
\text { bajas precipitaciones }\end{array}$} & Sequía & $\begin{array}{c}\text { Índice de precipitación (I), Índice de precipitación } \\
\text { estandarizado (SPI) y variación porcentual eventos } \\
\text { ENOS }\end{array}$ & 6,5 - MEDIA ALTA \\
\hline & Incendios forestales & $\begin{array}{c}\text { Pendiente, cobertura vegetal, sequía y régimen de } \\
\text { temperatura }\end{array}$ & 5,6 - MEDIA \\
\hline & $\begin{array}{l}\text { Degradación de sue- } \\
\text { los - Expansión de la } \\
\text { frontera agrícola }\end{array}$ & $\begin{array}{l}\text { Expansión de la frontera agrícola y ganadera, la } \\
\text { explotación del recurso forestal y la aridez del suelo. }\end{array}$ & 5,4 - MEDIA \\
\hline \multirow{2}{*}{$\begin{array}{l}\text { Eventos asociados a las } \\
\text { altas precipitaciones }\end{array}$} & Inundación & Mapas de pendientes y régimen de lluvias & $3,1-$ BAJA \\
\hline & Remoción en masa & $\begin{array}{l}\text { Pendiente, cobertura vegetal, régimen de lluvias, } \\
\text { uso del suelo y probabilidad sísmica (clasificación) }\end{array}$ & 5,8 - MEDIA \\
\hline \multicolumn{2}{|c|}{ Sismo } & Mapa del SGC (antes Ingeominas) & 7,0- ALTO \\
\hline
\end{tabular}

Fuente: elaboración propia

En cuanto a la evaluación de las amenazas se utilizaron índices e indicadores específicos para cada evento, además de los mapas de riesgos existentes como se puede apreciar en la Tabla 2. Siendo los eventos con mayor calificación las sequías, la remoción en masa y los sismos.

Respecto de los sismos, aceleradores de la remoción en masa y causantes de daños en infraestructura, se debe anotar que, la mayoría del área posee un relieve fuertemente ondulado (altas pendientes) y se encuentra afectada por el sistema de fallas inversas de la vertiente oriental de la cordillera, siendo la falla de Machetá la más importante en la región. De lo anterior se concluye que: las veredas La Junia, Tunjaque y Treinta y Seis están expuestas a una amenaza alta por sismo, debido a que se encuentran ubicadas sobre la franja de aceleración sísmica que oscila entre 200 y $250 \mathrm{~cm} / \mathrm{s} 2$ y la vereda de Jerusalén se encuentra ubicada en una franja de amenaza media con aceleración sísmica entre 150 a $200 \mathrm{~cm} / \mathrm{s} 2$. Aunque los sismos no representan un evento recurrente en las afectaciones a los servicios de acueducto y alcantarillado en Colombia ( $2 \%$ de 2.960 registros), si han representado grandes pérdidas para el sector (Mvct 2013). Por esto es importante tener en cuenta que, los acueductos veredales y las vías cercanas a cauces de quebradas y ríos no están construidos con normas sismoresistentes, sumado esto al hecho de que las redes de drenaje son mixtas (tubería-manguera), lo que las convierte en infraestructuras vulnerables a los embates propios de un sismo, situación que pone en riesgo la disponibilidad del recurso hídrico.

El análisis de la sequía se centró en el estudio de los índices de precipitación (I), de precipitación estandarizada (SPI) y la variación porcentual promedio de 
precipitación, específicamente para los periodos El Niño. Además de los índices se tuvo en cuenta el resultado de los balances hídricos en tiempo normal y bajo la incidencia del fenómeno Enos. Con este índice (I), los periodos en los que normalmente se reduce la precipitación en la cuenca del río Blanco se ubican en la categoría de meses muy secos el 11,6\% y en la categoría de precipitación por debajo de lo normal el 22,8\%, para el periodo 1997-2012. En los periodos de ocurrencia de El Niño, el índice (I) permitió detectar los meses específicos en los que hubo sequía en la región, es así que, El Niño 1997-1998 mostró un 57\% de meses en el rango de secos y muy secos. La posibilidad de estudio del SPI para diferentes escalas de tiempo permitió apreciar el inicio y final de temporadas de sequía, además de la identificación de meses aislados en los cuales la precipitación no fue suficiente. A partir de la escala de estudio SPI3, que refleja el comportamiento de la sequía para aplicaciones agrícolas (comportamiento que se correlaciona con el índice $\mathrm{Z}$ móvil), considerado como el mejor para evaluar la sequía agrícola, según Hurtado y Cadena (Hurtado \& Cadena 2002) incluido como índice para el análisis de sequías en la última versión del Estudio Nacional del Agua ENA 2014(Ideam 2015), se encuentra que: durante la ocurrencia de El Niño 97 - 98 hay señal de sequía durante el $47,9 \%$ del tiempo de ocurrencia del evento; de este porcentaje, el 19,8\% corresponde a meses moderadamente secos, el $12,4 \%$ a meses severamente secos y el $15,7 \%$ a meses extremadamente secos.

Considerando los resultados arrojados por los índices utilizados en el estudio, se pudo establecer un rango medio-alto de amenaza de sequía para las veredas en estudio. En el periodo seco de la cuenca, comprendido entre diciembre y la primera quincena de marzo se presentan muy pocas precipitaciones, lo que ocasiona escasez del recurso hídrico; la baja humedad y las altas temperaturas acentúan los efectos de la sequía aumentado la probabilidad de ocurrencia de incendios forestales. En estos meses, las bajas temperaturas al amanecer, debido a los cielos despejados en la noche, aumentan la probabilidad de heladas, lo cual pone en riesgo los cultivos. Todos estos efectos hidroclimáticos se intensifican con la ocurrencia del fenómeno El Niño.
Para el análisis de la remoción en masa se utilizaron indicadores como, las características litológicas y topográficas de la región y los agentes desencadenantes como el clima (fuertes y/o frecuentes lluvias), los sismos y las actividades antrópicas. El asentamiento de la población en zonas de ladera, así como el cambio de uso del suelo y la pérdida de cobertura vegetal debido a la actividad agrícola, son considerados parte de la problemática, situación que se ha ido incrementando con el tiempo en la región de estudio; la desnudez del suelo sumada a las condiciones geográficas (pendientes entre 7 y 25\%), las fallas geológicas y las condiciones climáticas, hacen que la remoción en masa sea considerada como una amenaza de nivel medio en la región. Cabe mencionar la alta probabilidad de que el nivel de amenaza aumente para los periodos en los que se presente el fenómeno de La Niña, esto como resultado del notorio incremento que muestran las lluvias (potenciadoras de los eventos de deslizamiento) cuando hace presencia este fenómeno.

El estudio de la vulnerabilidad global hídrica se hizo a partir de la ecuación 2, por lo cual fue necesario determinar primero la vulnerabilidad total en cada uno de sus seis componentes (ecuación 3). La vulnerabilidad del recurso hídrico para las veredas Treinta y Seis, Jerusalén, La Junia y Tunjaque se consideró como medio-alta debido, principalmente, a la notoria falta de recursos técnicos, humanos y socioeconómicos para hacer frente a los potencialmente catastróficos eventos (de origen natural o antrópico); la falta de recursos dificulta el mantenimiento y la ampliación de las redes de servicios y, de manera especial, la reserva y distribución del recurso hídrico.

Respecto a la vulnerabilidad ambiental, se encontró que esta se incrementa debido a: problemáticas relacionadas con la compactación y erosión de suelos debido a actividades agrícolas; la contaminación de fuentes hídricas debido al uso de pesticidas; la notoria disminución de la capa vegetal y las prácticas de incineración de residuos que contribuyen con la contaminación del aire (debido a la emisión de gases de efecto invernadero durante dicha práctica). En cuanto a la vulnerabilidad físico-estructural, se encontró que los acueductos veredales no cuentan con las estructuras 
de almacenamiento necesarias para asegurar las reservas de agua para las temporadas de sequía; también se determinó que el hecho de que las redes de distribución del agua sean mixtas aumenta la posibilidad de un evento de remoción en masa y, por consiguiente la vulnerabilidad de la población frente a este.

En cuanto al componente económico la población es vulnerable debido al escaso presupuesto entregado o destinado al mejoramiento de la infraestructura de servicios y salud, la gestión del riesgo de desastres y el cambio climático por parte del municipio de La Calera y los habitantes del sector. En el componente social inciden en la valoración de la vulnerabilidad el factor de educación, cultural y organización comunal respecto a la gestión del riesgo y el manejo de amenazas relacionadas a los efectos del cambio climático.

El análisis del factor de vulnerabilidad por recursos, deja ver que estos no son suficientes y algunos de los existentes no son los adecuados para que la población pueda hacer frente a los impactos que se derivan de la ocurrencia de eventos con características potencialmente catastróficas. Con respecto a este factor, las veredas en estudio fueron calificadas en un nivel de vulnerabilidad medio alta, situación que contribuye a la baja resiliencia (posibilidad de respuesta y recuperación) de la población. La escasa disponibilidad de recursos (humanos, técnicos y físicos, principalmente) está relacionada, entre otros, por la distancia de la zona de estudio respecto al casco urbano del municipio de La Calera.

En general, las veredas estudiadas poseen en la actualidad un nivel de riesgo de desabastecimiento hídrico medio, siendo Tunjaque la que presenta un mayor nivel, seguida de La Junia, lo anterior debido principalmente a que son las veredas con mayor actividad agropecuaria y en las que parece dársele la menor importancia a la conservación y manejo sostenible de los ecosistemas. En cuanto a los eventos adversos, la sequía y la remoción en masa fueron valorados como los de mayor riesgo de ocurrencia, quedando ubicados en un nivel medio alto, excepto en la vereda Jerusalén donde se determinó para estos un riesgo medio (resumen de resultados en la Tabla 3).
De los resultados encontrados, se puede decir que hay estrés del recurso hídrico en la zona cuando la demanda supera la oferta, en condiciones donde las posibilidades de resiliencia de la cuenca son muy bajas; estos momentos de déficit son notorios en épocas de ocurrencia de El Niño debido a las bajas precipitaciones y los altos niveles de evaporación que acompañan el evento. Es importante anotar, además, que la infraestructura de almacenamiento es muy pobre considerando las presiones de uso en el área de estudio, lo que hace muy vulnerable a la población frente a la posible disminución de la disponibilidad hídrica. Esta condición obliga a los habitantes de la región a desplazar su ganado hacia las fuentes de agua más cercanas; la falta del recurso en los hogares aumenta el lavado de ropas en las rondas de ríos y quebradas, situación que contribuye con la contaminación de estas fuentes hídricas a causa de la disposición de contaminantes, entre ellos las excretas y jabones empleados en el lavado.

Durante los meses de mayo, junio, julio y octubre, se aprecia un aumento notorio en las cantidades de lluvia, situación que se confirma con los índices de precipitación (I) y de precipitación estandarizada (SPI); durante este periodo son más frecuentes los meses considerados muy lluviosos o extremadamente húmedos; esto se comprueba, tanto para tiempo normal, como para épocas de ocurrencia de La Nińa. Es de anotar que las épocas de altas lluvias están acompañadas en muchos casos por eventos de deslizamiento, los cuales aumentan, paradójicamente, la vulnerabilidad de la población debido al incremento de la probabilidad de desabastecimiento hídrico. Esta vulnerabilidad está relacionada con la exposición de los acueductos en algunas de las veredas, ya que sus redes de distribución pueden verse afectadas por el incremento de la escorrentía y el movimiento o remoción de tierras, asociados a las fuertes lluvias. La escorrentía genera el lavado de laderas, con el que se da, no solo el desprendimiento y flujo de detritos, tierra y lodo, sino el escurrimiento de fertilizantes, plaguicidas y excretas de ganado, entre otros, que luego se depositan en ríos y quebradas contaminando sus aguas. 
El análisis prospectivo, relacionado con el riesgo futuro en la región de estudio para el periodo 2011-2040, bajo los escenarios de cambio climático A2 y B2 propuestos en su momento para el área de jurisdicción de la CAR (Pabón 2011), deja ver una tendencia al aumento de los posibles efectos debidos a la ocurrencia de los eventos considerados en la Tabla 3. Este aumento no presenta un cambio importante entre los escenarios A2 y B2 para el periodo 2011 - 2040 ya que, en términos generales, las diferencias sugeridas por Pabón para la región CAR en el comportamiento de variables como la precipitación (aumento en ambos escenarios del 10\%), humedad relativa (variaciones entre $-1 \%$ y $1 \%$, y hasta el $3 \%$ en el sector norte de la vereda de Jerusalén) y evapotranspiración (disminución del 10\% en ambos escenarios) muestran gran similitud (Pabón 2011).

Tabla 3

Calificación del riesgo futuro en el área de estudio.

\begin{tabular}{|c|c|c|c|c|c|c|c|c|c|c|}
\hline \multirow{3}{*}{ 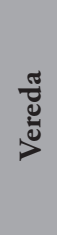 } & \multirow{3}{*}{\multicolumn{2}{|c|}{ Evento }} & \multicolumn{2}{|c|}{ Riesgo actual } & \multicolumn{6}{|c|}{ Riesgo futuro } \\
\hline & & & \multirow{2}{*}{$\begin{array}{c}\mathbf{R}= \\
\mathbf{F}\left(\mathbf{A}^{*} \mathbf{V}\right)\end{array}$} & \multirow[t]{2}{*}{ Nivel } & \multicolumn{2}{|c|}{$\begin{array}{c}\text { Factor de Ponderación } \\
\text { por Cambio Climático } \\
\text { FPCC }\end{array}$} & \multicolumn{2}{|c|}{$\begin{array}{c}\text { Riesgo Proyectado por } \\
\text { Escenarios de Cambio } \\
\text { Climático }\end{array}$} & \multicolumn{2}{|c|}{ Nivel } \\
\hline & & & & & A2 & B2 & A2 & B2 & A2 & B2 \\
\hline \multirow{5}{*}{ 宽 } & \multirow{3}{*}{$\begin{array}{l}\text { Eventos asociados a } \\
\text { bajas precipita-ciones }\end{array}$} & Sequía & 3,8 & M & 1,06 & 1,07 & 4,0 & 4,1 & $\mathrm{M}$ & $\mathrm{Ma}$ \\
\hline & & $\begin{array}{l}\text { Incendios } \\
\text { forestales }\end{array}$ & 3,0 & M & 1,06 & 1,07 & 3,2 & 3,2 & $\mathrm{M}$ & M \\
\hline & & $\begin{array}{l}\text { Degradación } \\
\text { de suelo }\end{array}$ & 2,5 & B & 1,06 & 1,07 & 2,7 & 2,7 & M & M \\
\hline & \multirow{2}{*}{$\begin{array}{l}\text { Eventos asociados a } \\
\text { altas precipita-ciones }\end{array}$} & Inundación & 2,3 & B & 1,07 & 1,08 & 2,5 & 2,5 & B & B \\
\hline & & $\begin{array}{l}\text { Remoción } \\
\text { en masa }\end{array}$ & 3,0 & M & 1,07 & 1,08 & 3,2 & 3,2 & M & M \\
\hline \multirow{5}{*}{ 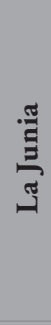 } & \multirow{3}{*}{$\begin{array}{l}\text { Eventos asociados a } \\
\text { bajas precipita-ciones }\end{array}$} & Sequía & 4,6 & $\mathrm{Ma}$ & 1,06 & 1,07 & 4,9 & 4,9 & $\mathrm{Ma}$ & $\mathrm{Ma}$ \\
\hline & & \begin{tabular}{|l|} 
Incendios \\
forestales
\end{tabular} & 3,8 & M & 1,06 & 1,07 & 4,0 & 4,1 & M & $\mathrm{Ma}$ \\
\hline & & $\begin{array}{l}\text { Degradación } \\
\text { de suelo }\end{array}$ & 3,9 & M & 1,06 & 1,07 & 4,1 & 4,2 & $\mathrm{Ma}$ & $\mathrm{Ma}$ \\
\hline & \multirow{2}{*}{$\begin{array}{l}\text { Eventos asociados a } \\
\text { altas precipita-ciones }\end{array}$} & Inundación & 2,1 & B & 1,07 & 1,08 & 2,2 & 2,3 & B & B \\
\hline & & $\begin{array}{l}\text { Remoción } \\
\text { en masa }\end{array}$ & 4,1 & $\mathrm{Ma}$ & 1,07 & 1,08 & 4,4 & 4,4 & $\mathrm{Ma}$ & $\mathrm{Ma}$ \\
\hline \multirow{5}{*}{ 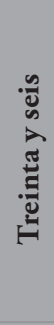 } & \multirow{3}{*}{$\begin{array}{l}\text { Eventos asociados a } \\
\text { bajas precipita-ciones }\end{array}$} & Sequía & 4,0 & M & 1,06 & 1,07 & 4,2 & 4,3 & $\mathrm{Ma}$ & $\mathrm{Ma}$ \\
\hline & & $\begin{array}{l}\text { Incendios } \\
\text { forestales }\end{array}$ & 3,7 & M & 1,06 & 1,07 & 3,9 & 4,0 & $\mathrm{M}$ & M \\
\hline & & $\begin{array}{l}\text { Degradación } \\
\text { de suelo }\end{array}$ & 3,5 & M & 1,06 & 1,07 & 3,7 & 3,7 & $\mathrm{M}$ & M \\
\hline & \multirow{2}{*}{$\begin{array}{l}\text { Eventos asociados a } \\
\text { altas precipita-ciones }\end{array}$} & Inundación & 1,4 & $\mathrm{~B}$ & 1,07 & 1,08 & 1,5 & 1,5 & $\mathrm{~B}$ & $\mathrm{~B}$ \\
\hline & & $\begin{array}{l}\text { Remoción } \\
\text { en masa }\end{array}$ & 3,9 & M & 1,07 & 1,08 & 4,2 & 4,2 & $\mathrm{Ma}$ & $\mathrm{Ma}$ \\
\hline \multirow{5}{*}{ : } & \multirow{3}{*}{$\begin{array}{l}\text { Eventos asociados a } \\
\text { bajas precipita-ciones }\end{array}$} & Sequía & 4,6 & $\mathrm{Ma}$ & 1,06 & 1,07 & 4,9 & 4,9 & $\mathrm{Ma}$ & $\mathrm{Ma}$ \\
\hline & & $\begin{array}{l}\text { Incendios } \\
\text { forestales }\end{array}$ & 4,1 & $\mathrm{Ma}$ & 1,06 & 1,07 & 4,3 & 4,4 & $\mathrm{Ma}$ & $\mathrm{Ma}$ \\
\hline & & $\begin{array}{l}\text { Degradación } \\
\text { de suelo }\end{array}$ & 4,0 & M & 1,06 & 1,07 & 4,2 & 4,3 & $\mathrm{Ma}$ & $\mathrm{Ma}$ \\
\hline & \multirow{2}{*}{$\begin{array}{l}\text { Eventos asociados a } \\
\text { altas precipita-ciones }\end{array}$} & Inundación & 2,2 & $\mathrm{~B}$ & 1,07 & 1,08 & 2,4 & 2,4 & B & $\mathrm{B}$ \\
\hline & & $\begin{array}{l}\text { Remoción } \\
\text { en masa }\end{array}$ & 4,2 & $\mathrm{Ma}$ & 1,07 & 1,08 & 4,5 & 4,5 & $\mathrm{Ma}$ & $\mathrm{Ma}$ \\
\hline
\end{tabular}

Nota: nivel de riesgo B: bajo (1,0-2,5), M: medio (2,6-4,0), Ma: medio alto (4,1-6,0), A: alto $(6,1-8,0)$ y MA: muy alto (8,1-10,0).

Fuente: elaboración propia 
El aumento del riesgo futuro para eventos asociados a bajas precipitaciones está relacionado con la variación de los parámetros ya mencionados y el aumento de la temperatura en el área de estudio (entre $2^{0}$ y $3^{0} \mathrm{C}$ para el escenario $\mathrm{A} 2$ y de $1^{\circ} \mathrm{C}$ para el escenario B2). Este calentamiento sería posible observarlo a lo largo del año, siendo el aumento más marcado desde mediados de noviembre hasta comienzos de abril, temporada en la que normalmente disminuye la nubosidad y las lluvias, debido al régimen monomodal que impera en la región, relacionado principalmente con las condiciones orográficas. Es en esta temporada cuando es más probable la sequía y, con ella, la ocurrencia de incendios forestales los cuales muestran, además, relación con acciones antrópicas como el corrimiento de la frontera agrícola. A pesar que los escenarios indican un aumento de más del 10\% en los totales de lluvia en la zona; se estima que los episodios de alta precipitación tenderán a producirse con menor frecuencia pero es probable que los pocos aguaceros sean más intensos; es bueno anotar que, según el Ipcc (2013) habrían periodos secos más prolongados entre episodios de lluvia

Este aumento en los valores que toma el riesgo futuro (ver Tabla III) relacionado con la sequía se verá reflejado en la disminución de la disponibilidad del recurso hídrico para el consumo humano, para el desarrollo de las actividades agropecuarias y para suplir las necesidades de los ecosistemas; estas condiciones traerán consigo el aumento del estrés hídrico y con ello el incremento del riesgo en la seguridad alimentaria y en los niveles de afectación de los recursos naturales. Estas situaciones, al igual que los eventos de origen socio-natural que las acompańan (incendios forestales y degradación del suelo, principalmente) se incrementarán de manera notoria durante la ocurrencia de El Niño.

Los escenarios A2 y B2 propuestos para la región CAR (Pabón 2011) sugieren un aumento de la precipitación en el área de estudio de más del 10\% para el periodo 2011 - 2040 en comparación con la precipitación actual, por esta razón en la valoración del riesgo futuro se pudo percibir un aumento en el número de eventos asociados a altas precipitaciones (inundación y remoción en masa). Esto significa que muy probablemente, a lo largo del periodo considerado para la proyección, se tendrá un aumento en la ocurrencia y severidad de eventos como los deslizamientos y avalanchas. Vale la pena anotar que, de acuerdo con las proyecciones dadas por el Ipcc (2013), es probable que aumenten los episodios de precipitaciones extremas, es decir, que sean más frecuentes los aguaceros intensos desencadenantes de crecidas en ríos de alta pendiente, inundaciones por desbordamiento y movimientos de tierra en las laderas. Se prevé, entonces, que todos estos fenómenos a los que se hace referencia ocurrirán con más frecuencia e intensidad durante los periodos en que se presente el fenómeno de La Niña.

Finalmente, se considera importante recordar que la región se caracteriza por poseer altas pendientes, las que acompañadas de altas precipitaciones hacen probable la ocurrencia de deslizamientos y avenidas torrenciales, situación que se asocia a las posibles restricciones en el suministro del agua, debido al impacto que sobre la red de distribución estos fenómenos pueden causar. Este riesgo de colapso de la red se vería multiplicado en caso de combinarse las lluvias y la degradación de las de las montańas con la ocurrencia de un sismo. Es por esta razón que estos eventos de origen geológico fueron tenidos en cuenta y analizados como fenómenos que al presentarse pueden potenciar la remoción en masa y contribuir con la probabilidad de daño y deterioro de la red de distribución del agua, así como de la red vial en la zona.

\section{Conclusiones}

Considerando la oferta hídrica en el área de la microcuenca río Blanco, donde se encuentran ubicadas las veredas de estudio Treinta y Seis, Jerusalén, La Junia y Tunjaque, el balance hídrico mostró déficit de precipitación respecto a la evapotranspiración real para los meses de diciembre a febrero y un superávit para los meses de mayo a julio. Se encontró, además que, para el periodo de estudio, 1997 - 2012, la 
cuenca río Blanco estuvo sometida a estrés hídrico moderado, lo cual permite suponer un aumento de la vulnerabilidad de los habitantes de la región y de los ecosistemas por desabastecimiento frente a la variabilidad y el cambio climático futuros.

En cuanto a la identificación de amenazas en las veredas estudiadas, fueron tenidos en cuenta los sismos (por lo expuesto de la infraestructura), la sequía y la remoción en masa como los eventos más amenazantes para el recurso hídrico y la población. La vulnerabilidad del recurso hídrico alcanzó el rango de medio-alta, debido principalmente a la notoria falta de recursos técnicos, humanos y socioeconómicos para hacer frente a la posible ocurrencia de eventos de origen natural o antrópico, considerados potencialmente catastróficos. Al combinar las características de las amenazas con la vulnerabilidad encontrada en el área de la cuenca se observó que, en general, las veredas allí ubicadas presentan un nivel de riesgo medio para desabastecimiento hídrico. Este riesgo se acentúa en las veredas de Tunjaque y La Junia, las cuales poseen mayor actividad agropecuaria y en las que no hay programas claros sobre el manejo y conservación de los ecosistemas.

Considerando el calentamiento global como factor de aumento de cambios de frecuencia y/o intensidad de fenómenos meteorológicos extremos asociados a la ocurrencia de desastres, se determinó el aumento del riesgo futuro de los eventos asociados a bajas precipitaciones como sequía, incendios forestales y degradación del suelo - corrimiento de la frontera agrícola, y aquellos relacionados con las altas precipitaciones como inundaciones y remoción en masa; el análisis se realizó considerando los escenarios de cambio climático A2 y B2 propuestos para la jurisdicción CAR y utilizando un factor de ponderación de cambio climático (Fpcc). Aunque los incrementos calculados no permitieron ver un cambio en el nivel del riesgo futuro para todos los eventos analizados, esto no significa que este incremento no sea importante para los cambios que se prevén en el futuro. Este aumento concuerda con los estimados para la temperatura $\left(1^{0} \mathrm{C}\right.$ para $\mathrm{A} 2 \mathrm{y}$, entre $3^{0}$ y $4^{0} \mathrm{C}$ para el escenario B2) y la precipitación (más del 10\% de precipitación en ambos escenarios) para la región, los cuales pueden impactar de manera drástica el régimen hidrológico y sus condiciones de calidad y uso.

Es necesario destacar que los escenarios empleados para este estudio no contemplan el aumento de la variabilidad climática, la cual según los informes del Ipcc 2007 y 2013 (Grupo I) el evento El Niño - La Niña - Oscilación Sur Enos, seguirá siendo el modo dominante del clima en el siglo XXI, con influencias globales, ocasionando que se intensifique la variabilidad de las precipitaciones regionales que induce. $\mathrm{Al}$ respecto es importante decir que el análisis de la vulnerabilidad y, por consiguiente, la determinación del riesgo para el área de estudio se realizó teniendo en cuenta las características de eventos ocurridos de variabilidad climática como, El Niño y La Nińa y su relación con los períodos de sequía y los tiempos de lluvias extremas.

Es así como se comprueba que el cambio climático constituye un gran desafío para la gestión integral del recurso hídrico, el cual se vería afectado en cuanto a disponibilidad, calidad y uso, además de su impacto sobre la salud, la seguridad alimentaria y la conservación de los ecosistemas, entre otros. Es por esto que es necesaria la incorporación de diferentes medidas de adaptación y mitigación, que sirvan como estrategias y oportunidades para garantizar el desarrollo sostenible y el bienestar de los habitantes de la región; que permitan, además, anticiparse, de manera proactiva para afrontar los desafíos generados por el cambio climático y la variabilidad climática natural.

\section{Reconocimientos}

Agradecimiento especial al Grupo Interno de Trabajo en Cambio Climático de la Corporación Autónoma Regional de Cundinamarca CAR, por el apoyo durante el desarrollo del trabajo. 


\section{Referencias}

Arango P 2012, 'Identificación y análisis de los factores de riesgo en el área de la cuenca alta del río Bogotá comprendida por los municipios de Zipaquirá, Cogua, Gachancipá y Nemocón’, Bogotá D.C.

Bates, B, Kundzewicz, Z, Wu, S \& Palutikof, J 2008, "El cambio climático y el agua”, Documento técnico del Grupo Intergubernamental de Expertos sobre el Cambio Climático, Secretaría del IPCC, Ginebra.

Guerrero, R \& S. K 2013, "Análisis sobre la capacidad de adaptación socioeconómica ambiental e institucional de la Subcuenca del Río Bajo-Ubaté, frente a los Escenarios de Cambio Climático propuestos por la Corporación Autónoma Regional de Cundinamarca y la Universidad Nacional”, Bogotá D.C., 2013.

Hurtado, G \& Cadena, M 2002, “Aplicación de índices de sequía en Colombia", Meteorología Colombiana, (5), 131-137.

Ideam 2010, "Estudio nacional del agua 2010", Instituto de Hidrología, Meteorología y Estudios Ambientales - Ideam, Bogotá D.C.

Ideam 2015, "Estudio nacional del agua 2014", Instituto de Hidrología, Meteorología y Estudios Ambientales - Ideam, Bogotá D.C.

Ipcc 2007, "Cambio climático 2007: informe de síntesis. Contribución de los grupos de trabajo I, II y III al cuarto informe de evaluación del grupo intergubernamental de expertos en cam- bio climático", Panel Intergubernamental de Expertos en Cambio Climático - Ipcc, Ginebra.

Ipcc 2012, "Special report - managing the risks of extreme events and disasters to advance climate change adaptation (Srex)", Ipcc, Cambridge, New York.

Ipcc 2013, "Climate change 2013: the physical science basis. contribution of working group i to the fifth assessment report of the intergovernmental”, Ipcc, Cambridge, New York.

López, V 2006, "Comportamiento histórico de la precipitación en la cuenca media y alta del río Tunjuelo y análisis del evento ocurrido entre los meses de mayo y junio de 2002", Épsilon, $7,7-22$.

Montealegre, E 2012, "Análisis de la variabilidad climática interanual (El Niño y La Niña) en la Región Capital, Bogotá Cundinamarca”, Observatorio Ambiental de Bogotá.

Mvct 2014, "Lineamientos de política de gestión del riesgo de desastres en la prestación de los servicios públicos de acueducto,alcantarillado y aseo", Ministerio de Vivienda, Ciudad y Territorio - Mvct, Bogotá D.C.

OMM 2012, "Guía del usuario sobre el índice normalizado de precipitación”, Organización Meteorológica Mundial - OMM, Ginebra.

Pabón, J 2011, "El cambio climático en el territorio de la Corporación Autónoma Regional de Cundinamarca", Universidad Nacional de Colombia - Corporación Autónoma Regional de Cundinamarca, Bogotá D.C. 


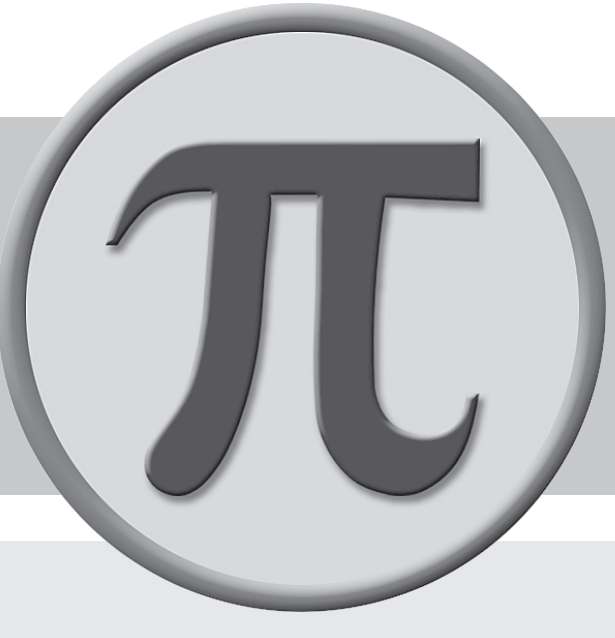


